\title{
6. Metafísica como lógica
}

Es bueno recordar que cuando Kant publicó su Crítica de la razón pura, se encontró con que sus contemporáneos no parecían haber comprendido su verdadero sentido, ya que lo interpretaban como una versión alemana de Berkeley. Por eso, con el propósito de hacerse comprender, escribe lo que cabría llamar un resumen de su propuesta crítica, y lo hace con el sugestivo nombre de Prolegómenos a toda metafisica futura que pueda presentarse como ciencia. Movidos por esa indicación, los idealistas alemanes, comenzando por Fichte, se propusieron entonces llevar a cabo la tarea que había iniciado Kant, tratando de elaborar esa metafísica bajo las condiciones establecidas por él.

En su distanciamiento frente al escepticismo, Kant había señalado cómo todo conocimiento pasa por la experiencia, aunque no todo provenga de ella. Ahora bien, si nuestro conocimiento debe someterse a las condiciones de la experiencia sensible, nunca podrá aspirar a conocer la totalidad como tal, ya que un conocimiento así tiene pretensiones de absoluto, y la experiencia marca al conocimiento de manera indefectible con el carácter de lo relativo.

Para responder a las exigencias de Kant, sin renunciar por ello al conocimiento metafísico, Hegel consideró necesario partir de la experiencia de la conciencia ordinaria para desarrollar todo un análisis ordenado de sus diversos comportamientos con respecto a la realidad, lo cual le permitía señalar 
al menos dos aspectos importantes. El primero, que las experiencias de la conciencia ordinaria muestran cómo la realidad se va desintegrando frente a la conciencia a medida que esta pretende conocerla, hasta el punto de convertirse, como sucede en la ciencia, en un "juego de fuerzas", es decir, en un proceso infinito de carácter dinámico en el que nada tiene consistencia propia, sino que todo se diluye en un incontenible estar pasando.

Es precisamente lo que nos muestra la Fenomenología del espiritu en sus tres primeras figuras, "certeza sensible, percepción y entendimiento". Comenzamos pensando que la realidad se halla compuesta de singulares únicos e irrepetibles, pero estos se nos convierten en "cosas", es decir, en entidades conectadas unas con otras en un entramado de relaciones que las determinan, para finalmente ser pensadas como meros "juegos de fuerzas" regidos por leyes, tal como las consideran las ciencias.

Refiriéndose a la forma como la filosofía comprende la realidad en cuanto devenir viviente, nos dice Hegel en el prólogo a la Fenomenología:

Así, lo verdadero es el delirio báquico en el que no hay miembro que no esté ebrio y, puesto que cada uno, en cuanto se separa, se disuelve igualmente de manera inmediata, él es igualmente la quietud transparente y simple. En realidad, ante el tribunal de ese movimiento no se sostienen las figuras singulares del espíritu, así como tampoco los pensamientos determinados, pero son también igualmente momentos positivos necesarios, tanto como negativos y desaparecientes (Fenomenología, 35).

La anotación es clara: la realidad se muestra como un devenir incontenible en el que los momentos pasajeros no dejan por ello de ser momentos reales y diferentes, pero inconsistentes, y lo mismo sucede con los conceptos que buscan comprender dicha realidad.

En ese devenir incontenible, en el que las cosas se muestran como momentos reales y a la vez pasajeros, la realidad como tal viene a mostrarse, entonces, 
como la posibilidad que se le ofrece a la conciencia para descubrirse a sí misma en su ser otro, para tratar de sacar a la luz, a partir de ese mundo informe y pasajero, lo que lo constituye como configurado y permanente, esto es, la estructura lógica que rige esa bacanal de la vida. Pero para ello tendrá que comprender que tal configuración racional de la realidad hunde sus raíces más allá del individuo, en la historia cultural que lo sostiene y dentro de la cual existe como tal. En otras palabras, quien filosofa deberá comprender que la configuración racional de lo real se halla condicionada por el proceso mismo mediante el cual la humanidad ha ido sacando a la luz tal configuración. Difícil encontrar un pensamiento que haya tenido más conciencia de su historicidad.

Esto explica las dos grandes partes que configuran el texto de la Fenomenología del espiritu. Una primera dedicada a examinar las experiencias de la conciencia individual en busca de la verdad (conciencia, autoconciencia y razón), y una segunda que analiza las experiencias de la conciencia colectiva como la raíz misma desde la cual la conciencia individual puede llegar a ser comprendida (espíritu, religión y saber absoluto).

El otro aspecto importante que descubre el análisis de le Fenomenología es que todo el conjunto de la experiencia humana, cuando se lo comprende en su ordenamiento racional, se halla sustentado en el hecho de que lo real posee la configuración de la conciencia misma. Esto quiere decir que la totalidad viene a manifestarse no solo como un proceso orgánico de autogeneración, sino más aún como un proceso espiritual. A este saber que comprende la estructura de lo real como idéntica a la estructura de la conciencia es al que Hegel denomina saber absoluto. Ahora bien, la diferencia entre un proceso meramente orgánico, como el que se da en una planta, y un proceso espiritual, es que en este último se cumple la idea de proceso en toda su plenitud. Para entenderlo mejor voy a detenerme para considerar lo que significa un proceso.

En su sentido ordinario, un proceso se da cuando algo deja de ser lo que es y se convierte en algo diferente. En ese sentido se supone que hay algo previo al proceso mismo que sirve de punto de partida, y que eso previo se desarrolla 
de tal manera que al final sigue siendo él mismo, pero habiéndose realizado en sus posibilidades. Sabemos que ya los griegos habían visto con claridad los problemas que presenta a la comprensión racional el concebir un proceso, es decir, un verdadero cambio; porque se trata de que la misma cosa es y no es la misma, tanto al comienzo del proceso como al final del mismo. Al comienzo, porque si la cosa ya fuera todo lo que debe ser, no podría tener lugar el proceso, de modo que la cosa debe no ser plenamente lo que ella debería ser. Y al final se dirá que la cosa es ahora plenamente, pero a condición de que sea de alguna manera la misma que era al comienzo. Porque si se tratara de que una cosa se convierte en otra por completo diferente, no tendríamos un proceso, sino una simple sustitución.

Ahora bien, lo que Hegel considera que debemos concebir no es simplemente que las cosas cambian y se transforman, sino que las cosas no son en realidad sino momentos pasajeros de un proceso absoluto; en otras palabras, que la realidad debe concebirse de tal manera que aquello que llamamos cosa no venga a ser sino solo un momento, es decir, una configuración transitoria que le otorga realidad al proceso mismo al desaparecer de inmediato. Ahora bien, al tratarse de la realidad como un todo, hay que pensar entonces que no existe algo previo al proceso como tal, y que si bien es cierto que los momentos van sucediéndose, ese mismo sucederse no implica que la totalidad como tal sea diferente a sí misma. Y la única manera de comprender esto es concebir la totalidad a imagen y semejanza de la conciencia. Porque es el "sí-mismo" (Selbst), como dice Hegel, es decir, ese carácter de mismidad que posee la conciencia, el que permite pensar un proceso en el que todo cambia para que todo permanezca igual, en el que lo mismo es a la vez otro de sí sin dejar de ser lo mismo, más aún, realizándose precisamente en ese su otro.

De ahí que, después de haber afirmado en el prólogo a la Fenomenología que todo el problema para comprender su filosofía consiste en concebir la sustancia también como sujeto, Hegel diga:

Además, la sustancia viviente es el ser que es en verdad sujeto, o, lo que significa lo mismo, que es en verdad real únicamente 
en cuanto ella es el movimiento del ponerse a sí misma, o la mediación del volverse otra consigo misma. Como sujeto, ella es la pura negatividad simple, y precisamente por ello es el desdoblamiento de lo simple o la duplicación contraponente, que es de nuevo la negación de esa diferenciación indiferente y de su contraposición; lo verdadero es únicamente esa igualdad que se restablece, o la reflexión en el ser-otro dentro de sí mismo, no una unidad originaria como tal, o inmediata como tal. Es el devenir de sí mismo, el círculo que presupone su finalidad como meta y la tiene como comienzo, y que solo es real mediante la ejecución y su fin (Fenomenología, 18).

Si comprender que la estructura de lo real es la misma estructura de la conciencia es lo que Hegel llama el saber absoluto, ese saber es absoluto, tanto porque con él se conoce lo absoluto mismo, aquello que es por sí y se conoce por sí, como definía Spinoza a la sustancia, como también porque se trata de un saber en el que lo sabido y el saber mismo se identifican. Ahora bien, si para llegar a comprenderlo Hegel considera que debe recorrerse el arduo camino de la Fenomenología, esto no significa que ese saber se deduzca de las experiencias de la conciencia, sino que la reflexión sobre las experiencias viene a mostrarnos que dicho saber se halla en la base misma de todas esas experiencias como su condición de posibilidad. Se trata, entonces, de una verdadera deducción trascendental de lo absoluto en los términos establecidos por Kant: el saber de lo absoluto viene a mostrarse como la condición de posibilidad de todo saber en general. La semejanza con la filosofía de Spinoza resulta así muy clara.

\section{La sustancia como sujeto}

Es bien conocido el texto de Hegel, que antecede al que acabo de citar, en el que nos dice de manera muy enfática: 
A mi modo de ver, que tendrá que justificarse por la exposición del sistema mismo, todo depende de aprehender y expresar lo verdadero, no como sustancia, sino igualmente como sujeto. A la vez, hay que observar que la sustancialidad incluye dentro de sí tanto lo universal o la inmediatez del saber, como aquella inmediatez que es ser o inmediatez para el saber (Fenomenología, 18).

Examinemos la segunda parte de esta cita, ya que señala con toda claridad que la idea de sustancialidad - y no cabe duda de que Hegel esté pensando en Spinoza, como lo confirman las consideraciones que hace a continuación- , que esa idea de sustancialidad implica no solo al pensamiento mismo, sino al ser o, en términos de Spinoza, al pensamiento y a la extensión. Y es precisamente a esa sustancia, que abarca tanto lo que es como al saber sobre lo que es, a la que Hegel considera que hay que concebir como sujeto.

Esta tesis, sin duda fundamental para comprender la metafísica que propone Hegel, ha sido objeto de muy diversas interpretaciones. Charles Taylor la ha sintetizado en forma muy clara:

Idealismo absoluto significa que nada existe que no sea la manifestación de la Idea, es decir, de la necesidad racional. Todo existe con un propósito, el de que la autoconciencia racional llegue a ser, y esto requiere que todo lo que existe sea la manifestación de la necesidad racional. Así, el idealismo absoluto se relaciona con la noción platónica de la prioridad ontológica del orden racional que subyace a la existencia externa, y al que la existencia externa se esfuerza por realizar, más bien que con la moderna noción post-cartesiana de dependencia con respecto a la mente que conoce (1979, p. 102).

Es claro que una concepción así resulta hoy muy extraña, y no han faltado quienes han tratado de ofrecer interpretaciones que se adecúen mejor a 
nuestra época "post-metafísica". Klaus Hartmann, en un escrito cuyo título mismo resulta muy diciente, Hegel: una visión no-metafisica, nos dice:

La filosofía de Hegel es una teoría de categorías, o de aquellas determinaciones de lo real que permiten reconstruirlo, y se confirman así como categorías. [...] Una categoría se "entiende", se explica o se justifica en términos de su función con respecto a hacer posible una ontología, es decir, satisfacer la razón (Hartmann 1971, p. 103).

Esto significaría que la propuesta hegeliana, lejos de ofrecer una metafísica, no estaría haciendo más que desarrollar los principios establecidos por la filosofía crítica kantiana, pero sin transgredir sus límites. Sin embargo, si abandonamos prejuicios empiristas que condenan todo intento de comprender la realidad como un todo a ser no más que simples elucubraciones mentales, y aceptamos nuestra capacidad de concebir el sentido mismo de lo que existe como un proceso absoluto, de modo que las condiciones de posibilidad de ese proceso vienen a ser las condiciones de posibilidad de lo que existe, tal vez la propuesta metafísica de Hegel no resulte tan desorbitada.

Spinoza estaba convencido de que podíamos lograr un concepto adecuado de sustancia que nos mostrara no solo la necesidad de su existencia, sino las determinaciones fundamentales de esta como determinaciones de todo lo que es o puede ser, así como las consecuencias que de ello se desprenden para la orientación de nuestro comportamiento. Lo que Hegel propone es dar un paso más en esa misma dirección y concebir esa sustancia, ya no solo como sustancia, sino igualmente como sujeto.

Tratemos entonces de comprender esta propuesta, a la que bien cabe considerar, tal como Hegel mismo lo indica, la clave para comprender su sistema: "A mi modo de ver, [...] todo depende de aprehender y expresar lo verdadero, no como sustancia, sino igualmente como sujeto" (Fenomenología, 18). Para ello resulta indispensable desarrollar tanto los aspectos metafísicos que ese principio comporta, como también los referentes al conocimiento, y 
reparar sobre todo en la trabazón ontológico-formal de los conceptos que lo articulan, tal como estos son presentados por Hegel en la Ciencia de la lógica.

En primer lugar, es conveniente precisar lo que significa comprender la sustancia como sujeto, porque, si se sigue la senda trazada por Kant, sujeto significa para Hegel aquella actividad mediante la cual se lleva a cabo la autorreferencia que caracteriza a la autoconciencia. Ahora bien, esa actividad autorreferencial no puede ser concebida como el acto de una entidad subyacente, de una "cosa pensante" (res cogitans), como la caracterizaba Descartes, sino como un obrar sin más, un obrar puro que se constituye a sí mismo en y por su mismo obrar. Pensar lo contrario sería negar de raíz la posibilidad de concebir la libertad, en cualquier sentido que pudiéramos darle a este término. Porque si pensamos al sujeto como un algo que subyace y antecede a la acción, ese algo solo podría comenzar a actuar mediante la intervención de algo otro que lo solicite a ello, y él no sería entonces la verdadera causa de su obrar.

Ahora bien, ese obrar y esa acción tienen además que desplegarse a través de momentos diferentes y diferenciables, lo cual nos lleva a tener que pensar la sustancia, es decir, aquello que es por sí, como el resultado del proceso mismo de autorrealización del sujeto. Tenemos así una razón más para entender por qué la sustancia no puede concebirse como el substrato que antecede a sus determinaciones, es decir, como aquello otro que se esconde tras ellas, como ese ignotum quid del que nos habla Locke, ya que de un tal sustrato solo cabría tener un concepto negativo. En este punto, Hegel avanza con paso firme por el camino abierto por Spinoza e impulsado por Fichte, al señalar que la verdadera sustancia tiene que ser concebida como causa sui, como proceso de autorrealización o de autoconstitución.

Por su parte, esas determinaciones que el sujeto va estableciendo con su obrar deben entenderse en su doble sentido de distinción frente a lo otro y de distinción con respecto a sí mismo, porque si bien es cierto que determinación es aquello por lo que una sustancia se distingue de otras sustancias, pensarla como sujeto que pone sus propias determinaciones, es decir, que se 
autodetermina, significa pensarla como estableciendo ella misma su propia distancia frente a lo otro y distinguiéndose ella misma de esa su determinación. Tenemos así que el sujeto que se determina frente a lo otro, se determina a la vez a sí mismo frente a su misma determinación al colocarse por encima de ella, es decir, al no confundirse con ella. A esa actividad de determinación, en cuanto es a la vez actividad de autodeterminación, la llama Hegel "actividad de la forma":

La sustancia pensada puramente como tal —nos explica Dieter Henrich - se halla dispensada de la actividad de la forma en ella. Pero en cuanto ella es a la vez sujeto, su forma se despliega a partir de su propia actividad (Henrich, 1987, p. 83)1.

Esto quiere decir que la sustancia pensada como pura sustancia habría que concebirla como una entidad subyacente, estática, diferente de su misma actividad, mientras que pensada como sujeto es ella misma su propio principio de determinación, es decir, se constituye como sustancia en cuanto resultado de su propia actividad. Ya la escolástica, buscando elaborar un concepto adecuado de sustancia, había propuesto concebirla como principium actionis, como principio de acción, a la manera como Leibniz, por su parte, llegó a concebir sus mónadas. Respecto a "El vocabulario de Leibniz" nos dice Martine de Gaudemar:

Lebniz no utiliza el término mónada en lugar de sustancia sino a partir de 1696. Este cambio de denominación corresponde a la implantación en la sustancia de un principio activo, llamado a veces entelequia. Este principio activo había hecho ya su aparición con otras apelaciones. Es "una cierta tendencia o fuerza

1 El desarrollo de lo que viene a continuación está inspirado en buena medida por el escrito de Dieter Henrich titulado La lógica hegeliana de la reflexión (Henrich, 1987, pp. 79-197). Me atrevería a decir que mi propósito ha sido introducir a la lectura de ese escrito de Henrich. 
primitiva para obrar, que es la ley inherente a esta sustancia y le ha sido impresa por el decreto de Dios" (2001, p.39).

La sustancia no es entonces pensada como un algo, como una cosa, sino como un impulso, como una fuerza o, más exactamente, como la fuente misma de la acción. Esto implica que la identidad de la sustancia concebida como sujeto no puede concebirse como distinta de su actividad de autodeterminación, sino que está constituida por esa misma actividad.

Con esto podemos avanzar hacia una consideración que habrá de ser definitiva para la concepción hegeliana del sistema como un todo, a saber, que si las determinaciones frente a lo otro y frente a sí misma por parte de la sustancia deben se concebidas como puestas por la acción de la sustancia misma, la relación que se establece con lo otro no puede ser pensada como una simple diferencia o diversificación, sino que corresponde a una verdadera oposición. El proceso de determinación, concebido así como un proceso de autodeterminación, debe entonces entenderse como un despliegue de oposiciones que se mantienen unificadas por la unidad misma del proceso mediante el cual la sustancia se despliega a sí misma como sujeto. Por eso, las oposiciones no son inteligibles sino como meros momentos de ese único proceso, y no pueden, por lo tanto, ser nunca datos últimos.

Vemos, entonces, por qué llama Hegel negatividad a esa "actividad de la forma", a saber, porque la subjetividad como concepto lógico es comprendida como el dinamismo que impulsa las contradicciones de un concepto, de manera que este, a través de ellas, alcanza la unidad superior que las reconcilia mediante el desarrollo de su propio significado. Este proceso de carácter conceptual o lógico tiene un orden y una necesidad de sus diferentes momentos, pero no tiene que ser concebido como un proceso de carácter temporal. Así, la negatividad como expresión de la subjetividad que es propia de la verdadera sustancia viene a ser el motor que impulsa el desarrollo lógico.

En esta forma, la sustancia que es sujeto cumple igualmente con los requisitos formales para desempeñar el papel de sujeto en la proposición predicativa, 
sin que para ello se la tenga que concebir como un substrato incognoscible. Al interpretar en esa forma, a partir de la teoría de la subjetividad, la función lógica como proceso de autodiferenciación del sujeto en sus predicados, nos dice Dieter Henrich que con ello Hegel "deja a un lado las maneras fundamentales de emplear las proposiciones predicativas. Es más bien la forma de la definición la que es presentada por él (de manera equivocada) como esa forma fundamental"' $(1987$, p. 85).

Pero tal vez Henrich no ha tenido en cuenta que Hegel comparte con Spinoza y con Leibniz un racionalismo radical para el que la distinción entre lo accidental y lo esencial no es en modo alguno una distinción definitiva. De esa manera, toda predicación, si es verdadera, tiene que ser en último término una forma de definición, y por ello, como el mismo Henrich lo señala luego, la idea de una sustancia como sustrato, como lo subyacente, como hypokeimenon, tiene que desaparecer, porque "en los predicados se despliega el significado del sujeto, y no queda nada del sujeto que, a la manera de lo puramente interior, de lo indeterminado o de lo en sí, pudiera sustraerse a la determinación por sus predicados" $(1987$, p. 85).

\section{EI sujeto como sustancia}

Pero si la sustancia debe concebirse como sujeto, ello no puede implicar que el concepto de sujeto sustituya sin más al de sustancia, de modo que cabe preguntar: ¿qué significa pensar el concepto de sujeto igualmente como sustancia? Y lo primero que debe responderse al respecto es que la autodeterminación mediante la cual el sujeto se despliega, lejos de poner en cuestión su identidad, debe, por el contrario, confirmarla y consolidarla. Si el sujeto debe concebirse como sustancia, es porque el proceso mediante el cual él pone sus propias determinaciones, establece sus diferencias frente a lo otro y afirma así su propia consistencia frente a la acción misma de determinar, es el mismo proceso mediante el cual él lleva a cabo su propia autoafirmación. En esta forma la sustancia, en lugar de ser vista como el punto de partida, como el presupuesto, como el origen y el fundamento inamovible, como la 
res cogitans, viene a ser comprendida más bien como el resultado, como la identidad que el sujeto va afianzando a través de sus diferenciaciones o, en palabras de Hegel, como "mismidad en la diferencia" o como "igualdad consigo mismo en la distinción”.

Vemos entonces cómo, al otorgarle un carácter sustancial al sujeto, se lo está distinguiendo de una subjetividad meramente formal y abstracta, para pensarlo como actividad que afirma y mantiene su propia identidad a través de sus mismas determinaciones cambiantes, y esto es así en la medida en que tales determinaciones son el resultado de su autodeterminación. Esto quiere decir que la sustancialidad del sujeto, lejos de ser un punto de partida, es concebida más bien como tarea, como propósito, lo cual expresa, dice Henrich, "que el sujeto debe sobrepasar su subjetividad unilateral y simplemente formal, para realizarse en relaciones determinadas a partir de la fuerza del sujeto que es negatividad" (1987, p. 91).

Concebir entonces a la realidad como sujeto y al sujeto como sustancia significa concebir la libertad, no como una abstracta indeterminación, como un puro libre albedrío neutro y ajeno al proceso de determinación, sino como la autodeterminación misma que, en el proceso de su progresiva diferenciación, consolida la plena identidad consigo.

\section{El análisis de la reflexión}

Creo que ahora podemos entender por qué, en la comprensión de la metafísica que propone Hegel, juega un papel primordial la articulación lógica que conecta la primera parte de su Lógica, titulada "Doctrina del ser", con la segunda, titulada "Doctrina de la esencia". Es allí, en ese paso o en esa articulación, donde el texto hegeliano por primera y tal vez única vez reflexiona de manera explícita sobre el concepto mismo de reflexión y sobre sus condiciones de posibilidad. 
Para entender esto mejor, considero importante recordar brevemente que la primera parte de la Lógica, la llamada "Doctrina del ser", examina aquellos conceptos mediante los cuales ordenamos y comprendemos los objetos tal y como nos son dados por la experiencia ${ }^{2}$. Lejos de toda consideración sobre el sujeto que los conoce, los objetos son tomados tal como se nos dan, y las categorías mediante las cuales los comprendemos vienen a corresponder, en lo fundamental, a las que ha empleado la metafísica tradicional anterior a Kant, cuyo punto de partida es el sentido común no reflexionado. El resultado de esa metafísica no es otro que la comprensión del encadenamiento necesario de todas aquellas determinaciones, tanto cualitativas como cuantitativas, mediante las cuales podemos pensar las diferencias de lo que simplemente es. Tal encadenamiento se mantiene a través de las determinaciones como el principio de medida o de mesura, que señala el equilibrio inestable que mantiene la totalidad en su constante cambio; equilibrio que, al verse trasgredido, vuelve por sus propios fueros. Cualidad, cantidad y mesura son así las tres grandes categorías que conforman la doctrina del ser.

El todo aparece así como un cosmos, como un orden inestable que se recupera siempre de toda desmesura, y que viene a ocupar el lugar del verdadero ser. Se muestra como el substrato de las relaciones mensurables y como la sustancia que se mantiene indiferente frente a la abigarrada y compleja diversidad de sus determinaciones. "La absoluta indiferencia — dice Hegel— es la última determinación del ser antes de volverse esencia" (Log. I, 397; esp. 334). Y este paso de la doctrina del ser a la doctrina de la esencia nos lo explica Xavier Zubiri en forma muy esclarecedora:

Para Hegel, "ser" es "tan solo ser", y decir de algo "solo" que "es", es nivelar todas las cosas: todas "son" y son "igualmente"; atendiendo tan solo al hecho de ser, todas tienen el mismo rango. La inmediatez es, por tanto, pura indiferencia (Gleichgültigkeit) y, por tanto, dispersión. En esto consiste la pobreza del

2 Estas consideraciones sobre la Lógica están inspiradas en el texto de George Noël (ver bibliografía de la parte I). 
ser. La razón concibe que esto 'no’ puede ser así. Repliega entonces (Reflexion) las notas sobre sí misma, las interioriza en cierto modo, esto es, las concibe como manifestaciones de una especie de núcleo interno de la cosa. Esto es la esencia (1963, p. 38).

Al seguir un análisis estrictamente conceptual, el paso de las categorías del ser a las categorías de la esencia se lleva a cabo en la Lógica a partir del ser como lo indiferente, como aquello que permanece siempre igual a través de todos los cambios. Pero conviene tener en cuenta que, si bien el proceso lógico sigue una necesidad de los conceptos mismos, los cuales, al mostrar su interna contradicción dan paso uno al otro, sin embargo, no se trata propiamente de una proceso deductivo en el que del concepto general de ser se fueran deduciendo, en una especie de prestidigitación conceptual, todas las categorías subsiguientes. Hay que fijarse, entonces, cuál ha sido la articulación lógica que ha llevado del concepto tradicional de ser a su nueva concepción como esencia, la cual inaugura, a partir de Descartes, la filosofía reflexiva propia de la modernidad.

Escuchemos el texto mismo de Hegel:

La absoluta indiferencia es la última determinación del ser antes de devenir esencia; pero ella no alcanza a la esencia. Se muestra como perteneciente todavía a la esfera del ser, en cuanto que, determinada como indiferente, tiene en ella a la diferencia como exterior, como cuantitativa. [...] Lo que aún falta aquí consiste en que esa reflexión no sea la reflexión exterior de la conciencia pensante y subjetiva, sino que la determinación propia de las diferencias de esa unidad sea la de suprimirse a sí mismas, con lo cual esa unidad se muestra así ser la absoluta negatividad, su propia indiferencia con respecto a sí misma, [es decir,] con respecto a su propia indiferencia, así como también con respecto a su ser otro" (Log. I, 397; esp. 334). 
En este denso párrafo se nos ofrece un completo resumen de lo que se propone pensar Hegel como el paso del concepto de ser al concepto de esencia. No es del caso entrar a analizar todos los detalles de este, ya que ello me exigiría extenderme demasiado. Voy a señalar únicamente cómo la dialéctica desarrollada por las categorías del ser desemboca, como se indicó anteriormente, en una concepción de este como el sustrato permanente e indiferente frente a la abigarrada multiplicidad de las determinaciones cualitativas y cuantitativas, a las que mantiene en un equilibrio inestable en tanto que medida o mesura ${ }^{3}$, es decir, como equilibrio entre lo cualitativo y lo cuantitativo. El ser es concebido así como lo eterno y permanente a través de todos los cambios y transformaciones y, como tal, se encarga de mantener en esos cambios el principio de la mesura o del equilibrio.

La diferencia es entonces pensada como algo que le es exterior al ser, como algo que, al fin de cuentas, le resulta indiferente. De este modo, lo que hace falta para que ingresemos de lleno en el pensamiento reflexivo de la Modernidad es introducir al sujeto para que se haga presente la reflexión como tal, pero ya no como la propia de una conciencia que contempla desde afuera, sino de tal manea que esas determinaciones o diferencias sean ellas mismas por sí mismas las que se suprimen y desaparecen en la unidad que se muestra como su ser otro. En otras palabras, comprender la desaparición de las diferencias en la unidad del ser indiferente es lo que nos lleva a comprender tanto el carácter reflexivo de esas diferencias como el del mismo ser que en esa forma se manifiesta como esencia.

Para comprende mejor el sentido de esta interpretación que hace Hegel del paso de una filosofía del ser a una filosofía de la esencia, o de una filosofía de la objetividad a una de la subjetividad, caracterizada por el llamado "giro copernicano", vale la pena examinar un momento cómo se constituye el concepto mismo de reflexión. Los pasos ya han sido señalados, y para comprenderlos conviene recordar que Hegel opera con un particular concepto de

3 En alemán el término Mass puede entenderse como "medida" o como "mesura". 
negación que no es la simple negación de algo, a la que él llama "negación abstracta". Se trata de la que él mismo llama "negación concreta", es decir, de aquella que, al negar algo, pone precisamente mediante esa negación lo otro de ese algo negado. Se trata en verdad de pensar el concepto de "alteridad", de lo otro como lo otro de lo uno, que es el concepto propio para comprender un proceso: algo pasa a ser algo otro, pero eso otro es $s u$ otro, de modo que en él no se pierde sino que se realiza.

La propuesta viene a ser así la de pensar esa indiferencia en la que consiste el ser como lo otro, pero no simplemente como lo otro de esto o de aquello, ya que estos últimos solo vienen a ser momentos insignificantes que terminan negándose a sí mismos para permitir que el ser sea; sino pensar esa indiferencia como lo simplemente otro, como la alteridad absoluta, es decir, como lo otro de sí mismo. Tal vez no resulte extraño que Hegel vea en ello la explicación lógica del agnosticismo que caracteriza a la Modernidad, tal como lo encontramos expresado en la filosofía de Kant. Lo absoluto, el ser, es concebido como lo simplemente otro, y esto, en primer lugar, significa aquello que no tiene ningún otro frente a sí, aquello que debe ser pensado de tal manera otro que nada tiene en común con nada, de modo que viene a situarse por fuera de toda posible alteridad. Así, al llevar hasta el extremo el pensamiento de la alteridad, hemos llegado hasta su misma negación, de modo que eso absolutamente otro tendrá que ser pensado como lo absolutamente negativo, no lo negativo de algo otro, sino como lo negativo de sí mismo.

Esta forma de pensar realmente extraña, cuya dificultad es reconocida por el mismo Hegel, viene a contradecir nuestra manera habitual de operar con el concepto de alteridad o de negación, pero podemos encontrarla esbozada en un pensador como Spinoza cuando elabora su concepto de atributo, y se halla presente también en la idea de "cosa en sí" kantiana. En ambos casos, por extraños que parezcan entre sí, se está buscando comprender la necesidad inherente al ser mismo de salir de sí, de extrañarse con respecto a sí mismo, sin lo cual no se ve cómo sea posible pensarlo de manera dinámica. Hay que dejar de pensarlo como lo puramente estático, ya que pensarlo así termina por despojarlo de todo sentido y valor. Si el ser mismo no es pensado como 
exteriorización (Entäusserung) o como "expresión", ${ }^{4}$ entonces lo finito, como lo otro del ser, vendría a perder toda consistencia ontológica, vendría a ser pura apariencia (Schein) y no verdadero fenómeno (Erscheinung).

Así pues, la propuesta que nos hace Hegel es la de pensar la reflexión del ser sobre sí mismo como una negatividad autorreferida, para conciliar así dos conceptos de muy diverso origen. Por una parte, el concepto heracliteano de devenir como unidad indisoluble de negación y afirmación, de ser y nada, interpretándolo como la afirmación que resulta de una negación de la negación; y, por otra, el concepto cristiano de Trinidad, como procesión de tres momentos en el seno de la absoluta unidad, lo que permite pensar un proceso eterno y un Dios que se autodetermina.

El inicio de la doctrina de la esencia se propone, entonces, comprender este nuevo concepto de ser desde la perspectiva de la reflexión, anunciando su propósito de manera clara y simple:
El ser es lo inmediato. En cuanto el saber desea conocer lo ver- dadero, lo que el ser es en y para sí, no se queda en lo inmediato y en sus determinaciones, sino que penetra a través de él con el presupuesto de que detrás de ese ser hay algo otro que el ser mismo, y de que ese trasfondo constituye la verdad del ser ( $\log$. II, 2; esp. 339).

La cita es clara y muestra lo que sucede inevitablemente cuando pretendemos comprender la realidad y no nos contentamos con referirnos a ella de manera pragmática. La reflexión de la conciencia tiene como consecuencia que la realidad misma se desdobla; en otras palabras, cuando reflexionamos sobre la realidad, esta misma se desdobla en lo que aparece y lo que verdaderamente es. Hegel está buscando comprender y superar, o comprender para superar, una concepción abstracta y contradictoria de esencia que la piensa

4 Para utilizar el término con el que Gilles Deleuze analiza la metafísica de Spinoza en Spinoza y el problema de la expresión. 
como aquello otro del ser que sin embargo viene a ser la verdad del mismo. Y para superar esta contradicción, de que algo tenga su verdad en algo otro que se muestra diferente de él, hace falta comprender de manera adecuada el proceso de reflexión o de desdoblamiento que se ha llevado a cabo en el concepto mismo de ser como resultado del análisis realizado en la primera parte de la Lógica.

Un primer intento va a consistir en interpretar el desdoblamiento como simple, de modo que tendríamos dos especies de seres, los esenciales y los noesenciales; pero con ello no se resuelve nada, ya que la atribución de entidad a unos u otros tendría que hacerse con relación a algo exterior al ser mismo. En efecto, si entendemos el desdoblamiento en su sentido más simple, como suele hacerlo el sentido común, la cuestión vendría a reducirse a tratar de distinguir en las cosas lo que tienen de esencial de lo que tienen de inesencial. Pero la pregunta sería entonces: ¿cuál es el criterio para esta diferenciación? Se estaría tratando de dividir el mundo en dos, uno compuesto por lo esencial y otro por lo inesencial, sin disponer de un criterio válido para ello; una especie de maniqueísmo conceptual, pero sin poder establecer un criterio válido para establecer cuál es el "bueno" y cuál el "malo", es decir, cuál es el esencial y cuál el inesencial. Ojo, porque al criticismo kantiano le sucede algo de esta especie; y también le sucede a Leibniz, para quien todos los predicados de un sujeto terminan siendo esenciales.

Sin embargo, este primer intento de comprender la reflexión nos deja un elemento positivo, y consiste, dice Hegel, en pensar el ser como "lo negativo puesto como lo negativo", es decir, en atreverse a pensar con toda seriedad la dialéctica del ser mismo: lo que se muestra como ser no es verdad sino mera apariencia, tesis que nos resulta bien conocida desde Platón, y que Hegel la encuentra en la raíz de todo escepticismo con respecto a la metafísica, pero también de todo idealismo. Solo que este último no entiende la realidad pasajera como mera apariencia, sino como verdadero fenómeno. No resulta por ello extraño que tanto el escepticismo como el idealismo se encuentren en las raíces mismas del proyecto cartesiano y vayan a marcar juntos la filosofía crítica de Kant. 
No se trata, por lo tanto, de pensar el ser como lo absolutamente otro, en el sentido de hallarse en un ámbito inalcanzable para nuestro conocimiento, porque precisamente este es el género de solución que muchos han intentado en vano. La propuesta de Hegel es reflexionar más bien sobre la misma reflexión para alcanzar un concepto adecuado de ella y comprender qué es lo está ocurriendo. Para lo cual será necesario llevar a cabo una verdadera inversión en el orden y el sentido de los conceptos complementarios de identidad y diferencia, de inmediatez y mediación, de igualdad-consigo y distinciónfrente-a-lo-otro. Comprender la esencia como reflexión significa comprender que la identidad, la inmediatez y la igualdad-consigo deben ser comprendidas como resultados de la diferenciación, de la mediación y de la distinción-frente-a-lo-otro, y no como concepto originarios:

La esencia es reflexión — dice Hegel—, el movimiento del devenir y del pasar que permanece dentro de sí mismo, en el cual lo distinto simplemente es determinado únicamente como negativo en sí, como apariencia (Log. II, 13; esp. 349).

Para ayudarnos a comprender ese puro devenir en sí mismo, Hegel no escatima las más osadas formulaciones: "El devenir en la esencia — nos dicemovimiento reflexionante es por lo tanto el movimiento de nada a nada y por ello el retorno a sí mismỏ (Log. II, 13; esp. 349), y un poco más adelante añade:

El ser es únicamente en tanto que el movimiento de nada a nada, y es así la esencia; y esta no tiene dentro de sí este movimiento, sino que es ese movimiento en tanto que la absoluta apariencia misma, la pura negatividad que no tiene nada fuera de sí que la haya negado, sino que niega únicamente lo negativo suyo, el cual es solo en ese negar (Log. II, 14; esp. 350).

Si tenemos en cuenta que el devenir es un eterno estar pasando que ya no tiene lo pasado ni tampoco tiene todavía lo futuro (de nada a nada), sino que es un eterno presente absolutamente pasajero, creo que la formulación de Hegel no debería extrañarnos. Con esta nueva concepción del ser como esencia, el 
texto procede a concebir el doble concepto de unidad que resulta necesario para pensar una verdadera unidad entre la unidad misma y la diferencia; no simplemente la unidad $y$ la diferencia, sino la unidad de unidad $y$ diferencia como momentos de la unidad misma. Para eso lleva a cabo lo que Henrich ha llamado un desplazamiento del significado, que consiste en pensar la inmediatez y la unidad no como punto de partida de la reflexión, sino como resultado de la negación auto-referida o de la negación que se niega a sí misma.

Esto le permite obtener dos conceptos de lo mismo que no por referirse a lo mismo pueden ser identificados, ya que representan dos momentos diferentes de un proceso único. El primer concepto es el de la identidad simple o la inmediatez, pero como simple posibilidad puesta por la inmediatez final, es decir, por la inmediatez mediada, la inmediatez del resultado. En otras palabras, en un verdadero proceso es el resultado el que determina el comienzo, ya que en ese comienzo debe hallarse el resultado como meta, como propósito o como fin. Esto lleva a Hegel a examinar tres momentos diferentes del proceso reflexivo, a los que determina con los términos de reflexión ponente, reflexión extrínseca y reflexión determinante.

En el primero, la "reflexión ponente", el acto reflexivo pone su resultado como punto de partida para negarlo y recuperarlo luego como resultado final. Esta formulación resulta comprensible si pensamos que todo verdadero proceso, en el que algo llega a ser más él mismo precisamente en cuanto deja de ser lo que era para volverse otro, implica poner el resultado o eso otro como el comienzo, pero con el carácter de lo posible, es decir, ponerlo como comienzo para negarlo y poderlo recuperar así como resultado. De esa forma, el resultado es puesto al comienzo como lo inmediato, como lo que solo es en tanto que posibilidad. Todo proceso implica, así, determinar un punto de partida que como tal solo tiene sentido en virtud del resultado final hacia el cual está referido.

Ahora bien, si la reflexión pone necesariamente eso otro para negarlo como tal, ello indica que posee un segundo momento, el de exterioridad con respecto a sí misma o de "reflexión extrínseca", la cual indica que eso que ha 
sido puesto como comienzo no solo debe ser negado por la reflexión, sino que debe negarse a sí mismo en virtud de su propia negatividad, debe exteriorizarse. Al haber sido puesto como lo posible, deberá salir de sí mismo, negarse, para dejar de ser posible y convertirse en real. Este momento es crucial para comprender lo que Hegel se está proponiendo. Tratemos entonces de formularlo en término menos abstractos o especulativos. Comprender el ser como reflexión significa comprender que lo finito no solo es puesto y negado por lo infinito como momento de la propia constitución de lo infinito, sino que lo finito mismo, en virtud de su intrínseca negatividad que lo distingue de lo infinito, se niega a sí mismo para identificarse así con lo infinito.

De esta manera, la reflexión como ponente y como extrínseca, es decir, en cuanto afirma su desdoblamiento y lo niega, pero lo niega en virtud de la negatividad propia de los momentos desdoblados, permite hablar de una "reflexión determinante", la cual, como dice el texto, "es simplemente la unidad de la reflexión ponente y de la extrínseca” (Log. II, 20: esp. 355). Si la reflexión implica un diferenciarse de los momentos y un identificarse de estos, la reflexión determinante no hace otra cosa que unificar esos dos procesos como momentos de un único proceso. De esta manera considera Hegel poder explicar el carácter inconsistente, pero a la vez indestructible, de lo que llamará a continuación las determinaciones de la reflexión. Se trata de todas aquellas categorías mediante las cuales el proceso reflexivo busca comprender una realidad que se muestra ella misma como proceso. En efecto, esos pares de categorías que elabora la reflexión (sustancia-accidente, fundamento-fundado, causa-efecto, etc.) poseen ese doble carácter que les es tan peculiar, a saber, que cada uno de los términos lleva en sí mismo la totalidad de la relación, a la vez que se contrapone al otro como su opuesto en una especie de reflejo especular.

Con este análisis global del paso de la lógica del ser a la lógica de la esencia he buscando comprender lo que Hegel considera el núcleo ontológico del pensamiento moderno, de ese pensamiento para el cual la metafísica termina convirtiéndose en una serie de contradicciones incontrolables mediante las cuales, como lo ha formulado Marshall Bermann citando a Marx, "todo lo 
sólido se desvanece en el aire". Pero allí mismo podemos detectar el germen conceptual que hará posible repensar la relación entre infinito y finito, unidad y multiplicidad, autorreferencia y diferencia-frente-a-lo-otro como elementos que pueden integrarse de tal manera que el primero de los términos (infinito, unidad, autorreferencia) pueda desempeñar la doble función de elemento de la contraposición y de unidad de los contrapuestos, es decir, servir de punto de partida y de punto de llegada de un proceso reflexivo o de autodesdoblamiento.

Con ello solo he podido esbozar la interpretación que nos ofrece Hegel del proceso conceptual mediante el cual se ha llevado a cabo la crítica a la metafísica tradicional y se han creado las condiciones para la elaboración de una nueva metafísica. Esta última solo podrá llevarse a cabo en la medida en que, superando desde su interior el proceso mismo reflexivo, se elabore un nuevo concepto de ser que ya no esté contrapuesto al concepto de esencia, sino que configure con este una verdadera unidad dinámica. Pero cabe decir que todos los elementos están dados y solo falta desarrollarlos: se trata, como nos había dicho el prólogo a la Fenomenología, de concebir la sustancia de Spinoza con las características del sujeto hegeliano, lo que implicará no solo desarrollar toda la lógica del concepto, es decir, de esa nueva concepción de sujeto como la forma adecuada de concebir lo que es, sino de desplegar todas sus potencialidades. Esto le permitirá a Hegel mostrar, más allá de la Lógica, cómo todo el mundo físico, la llamada Naturaleza, puede ser comprendida como la exteriorización de ese sujeto absoluto en la espacio temporalidad, y mostrar luego cómo el mundo humano o el mundo del Espíritu viene a ser el proceso de retomar esa Naturaleza desde su exterioridad para convertirla en un mundo humano, es decir lógico. Estas serán las tareas que deberá desarrollar el sistema mismo, tal como aparece en la Enciclopedia de las ciencias filosóficas. 